\title{
Expression of programmed cell death 5 protein inhibits progression of lung carcinoma in vitro and in vivo via the mitochondrial apoptotic pathway
}

\author{
SHINING XU*, GANG SUI*, LEI YUAN and ZHIQIANG ZOU \\ Department of Thoracic Surgery, The General Hospital of Jinan Military Command, \\ Jinan, Shandong 250031, P.R. China
}

Received November 16, 2013; Accepted April 28, 2014

DOI: $10.3892 / \mathrm{mmr} .2014 .2454$

\begin{abstract}
Lung cancer is one of the most common and serious types of cancer, and is characterized by uncontrolled cell growth and metastasis from lung tissues to other body parts. The programmed cell death $5(\operatorname{Pdcd} 5)$ protein is known to accelerate apoptosis in different cell types of tumor. The aim of the present study was to explore the role of Pdcd5 in lung carcinoma and to identify the mechanisms underlying the antitumorigenic properties of Pdcd5 in lung cancer. First, we detected and compared the expression of Pdcd5 in healthy and highly differentiated adenocarcinoma lung tissues. The results of histochemical staining and western blot analysis demonstrated that Pdcd5 expression is markedly decreased in highly differentiated lung adenocarcinoma. Next, we used the lung adenocarcinoma cell line A549 to study the effects of Pdc5 expression on proliferation and colony formation. The results revealed that the expression of Pdcd5 significantly inhibits cell proliferation and colony formation in A549 cells. Importantly, Pdcd5 expression induced tumor cell apoptosis, and the apoptotic proteins caspase- 3 and -9 were activated. The expression of B-cell lymphoma 2 (Bcl-2) was reduced and that of Bcl2-associated $\mathrm{X}$ protein (Bax) was increased, overall suggesting that the intrinsic apoptotic pathway is activated. Furthermore, using a mice xenograft model and vectors for stable expression or silencing of $P d c d 5$, we showed that stable expression of the protein significantly increases the survival rate of mice in vivo $(\mathrm{P}<0.01$ compared to control). In conclusion, both in vitro and in vivo experiments demonstrated that Pdcd5 expression inhibits proliferation and induces apoptosis in the A549 cell line, indicating that the Pdcd5 protein may
\end{abstract}

Correspondence to: Dr Zhiqiang Zou, Department of Thoracic Surgery, The General Hospital of Jinan Military Command, No. 25 Shifan Road, Jinan, Shandong 250031, P.R. China

E-mail: zouzhiqianghope@163.com

*Contributed equally

Key words: programmed cell death 5, lung cancer, apoptosis, caspase play an important role in the progression of lung cancer. Therefore, Pdcd 5 may be a promising target for the therapy of lung carcinoma.

\section{Introduction}

Lung cancer is the most common disease worldwide, with high incidence and mortality (1-3). Until 2008, an estimated 1.61 million new cases were reported, representing $12.7 \%$ of all new cancer types (4-6). The mortality rate (1.38 million cases) was estimated at $8.2 \%$ of the total mortality due to cancer, which renders lung cancer the most common type of cancer associated with mortality. Only in China, lung cancer has been ranked the number one cause of death among people with malignant tumors $(7,8)$. The registered mortality caused by lung cancer has increased by $464.84 \%$ in the past 3 decades (9). It has been reported that imbalance between cell proliferation and apoptosis plays a vital role in the development of lung cancer, along with mutations in tumor suppressor genes and oncogenes and inactivation of multiple genes (10-13).

The programmed cell death 5 ( $P d c d 5)$ gene, formerly designated as TF-1 cell apoptosis-related gene 19 (TFAR19), is involved in cell death and is upregulated during apoptosis (14). The gene was first cloned by the Peking University Center for Human Disease Genomics in 1999. It is expressed in more than 50 tissues in adult humans, and is highly expressed in tissues such as heart, kidney, adrenal gland, testis and placenta (15). The Pdcd5 protein translocates rapidly from the cytoplasm to the nucleus and plays an important role in the inhibition of the proteasome-dependent degradation of lysine acetyltransferase 5, which is involved in transcription, DNA-damage response and cell-cycle control. Disorders in the expression of PDCD5 have been associated with tumorigenesis $(16,17)$. Reduced Pdcd5 expression has been reported in several types of tumor and has been associated with the progression and prognosis of cancer. The protein showed potent antitumor activity via the interaction with the histone acetyltransferase Tip60 and the promotion of DNA damage-induced apoptosis $(16,18)$. However, the expression status and clinical significance of Pdcd5 in lung cancer, and whether Pdcd5 can efficiently inhibit the progression of lung carcinomas, have not yet been studied. 
In the present study, we compared the expression level of Pdcd5 in lung carcinoma and healthy lung tissues by immunohistochemistry and western blotting. We further explored whether the antitumor activity of Pdcd5 is regulated by the mitochondria-related apoptotic pathway. This study provides new perspectives for the early diagnosis, treatment and prognosis of lung cancer.

\section{Materials and methods}

Cell cultures and transfection. A549 cells were cultured in Dulbecco's modified Eagle's medium supplemented with $10 \%$ fetal bovine serum, $2 \mathrm{mM}$ L-glutamine, $100 \mathrm{U} / \mathrm{ml}$ penicillin, and $100 \mathrm{mM}$ streptomycin, in an atmosphere containing $5 \% \mathrm{CO}_{2}$. The PCI-neo-Pdcd5 plasmid was kindly provided by Dr Zhigang Liu (General Hospital of Jinan Military Command, Jinan, Shandong, China) and was transfected into the cells using Lipofectamine 2000 (Invitrogen, Carlsbad, CA, USA), according to the manufacturer's protocol. Pdcd5 short hairpin RNA (shRNA) lentiviral particles were purchased from Santa Cruz Biotechnology, Inc. (Santa Cruz, CA, USA).

Immunohistochemical analysis. Immunohistochemical staining was performed as described earlier $(19,20)$. Briefly, 30 highly differentiated lung adenocarcinoma tissues and 20 healthy lung tissues adajacent to these were fixed in $4 \%$ paraformaldehyde for $24 \mathrm{~h}$. The specimens were obtained from the Department of Thoracic Surgery, General Hospital of Jinan Military Command. The subjects and their families provided written informed consent prior to the study. Our study was performed in compliance with the Declaration of Helsinki and we obtained approval for the study from the Ethics Committee of The General Hospital of Jinan Military Command. The tissues were cut from paraffin blocks in 2-5 $\mu \mathrm{m}$ thick sections using a microtome (Microm HM 310, Microm International $\mathrm{GmbH}$, Walldorf, Germany), and were mounted on SuperFrost Plus slides (Carl Roth GmbH, Karlsruhe, Germany). The primary rabbit anti-human polyclonal antibody targeting Pdcd5 (1:100 dilution; Proteintech, Chicago, IL, USA) was incubated overnight in a moist chamber at room temperature. The secondary antibody, goat anti-rabbit, biotinylated antiIgG (Vector Laboratories Inc., Burlingame, CA, USA) was used at a 1:500 dilution. Paraffin-embeeded stained sections were observed under a light microscope (NAZAR AM5, Germany).

MTT assay. The MTT assay was performed as previously described (21-23). Briefly, A549 cells were placed into 48-well plates. Following cell adherence, the cells were transfected with the PCI-neo-Pdcd5 plasmid or Pdcd5 shRNA lentiviral particles for 24, 48 and $72 \mathrm{~h}$. The proliferation of A549 cells was determined by measuring the optical density (OD) of the samples at $570 \mathrm{~nm}$.

Colony formation assay. For the colony formation assay, cells were seeded in 6 -well plates $\left(2 \times 10^{3}\right.$ cells/well $)$ and transfected with PCI-neo-Pdcd5 or Pdcd5 shRNA for $24 \mathrm{~h}$. The medium was changed every two days, and the cells were cultured for ten days after transfection of Pdcd5. Surviving colonies ( $\geq 50$ cells/colony) were fixed with methanol, stained with
$1.25 \%$ crystal violet and counted under the light microscope ??h after transfection and for a total of ?? h.

Detection of apoptosis by fluorescence-activated cell sorting (FACS). A549 cells were trypsinized, washed three times with cold phosphate-buffered saline, and resuspended in $200 \mu \mathrm{l}$ binding buffer. Fluorescein isothiocyanate (FITC)-conjugated Annexin V (Biosea Biotechnology Co., Ltd., Beijing, China) was added according to the manufacturer's protocol, to a final concentration of $0.5 \mu \mathrm{g} / \mathrm{ml}$. Next, $1 \mu \mathrm{l}$ of $100 \mu \mathrm{g} / \mathrm{ml}$ propidium iodide working solution was added for incubation. Then, cells were incubated for $20 \mathrm{~min}$ at room temperature in the dark, and $400 \mu \mathrm{l}$ of binding buffer (5X Annexin binding buffer; $50 \mathrm{mM}$ HEPES, $700 \mathrm{mM} \mathrm{NaCl}, 12.5 \mathrm{mM} \mathrm{CaCl}_{2}, \mathrm{pH} \mathrm{7.4}$; Life Technologies, MA, USA) was added. The samples were immediately analyzed on a FACSCalibur flow cytometer (BD Biosciences, Franklin Lakes, NJ, USA).

Western blotting. Protein samples were prepared and separated by polyacrylamide gel electrophoresis as previously described (24-26). We used primary antibodies targeting Pdcd5, caspase-3 and -9, Bcl2-associated X protein (Bax), B-cell lymphoma $2(\mathrm{Bcl} 2)$, and $\beta$-actin (used as the loading control) at dilutions 1:3,000, 1:5,000, 1:5,000, 1:3,000, 1:5,000 and 1:10,0000, respectively. As a secondary antibody, we used the horseradish peroxidase-conjugated goat anti-mouse anti-IgG. All antibodies were purchased from Santa Cruz Biothechnology, Inc.

In vivo tumor xenograft study. BalB/c mice were purchased from the Experimental Animal Center of Shandong Medical University (Jinan, Shandong, China) and kept in a pathogenfree environment with a 12-h light/dark cycle. All experiments were conducted in conformation to the Guidelines of the Animal Care and Use Committee of the General Hospital of Jinan Military Command. A549 cells $\left(5 \times 10^{5}\right)$ were subcutaneously injected into the back of the mice. The mice were randomly divided into three groups $(n>5)$ : control (injected with untransfected A549 cells), Pdcd5 shRNA (injected with A549 cells transfected with the Pdcd5 shRNA) and PCI-neo-Pdcd5 (injected with A549 cells transfected with the PCI-neo-Pdcd5). The survival of mice was recorded daily and the survival rate was determined as $100 \mathrm{x}$ (number of survivors/total number of mice).

Statistical analysis. All the experiments were performed and repeated at least three times. The data were analyzed by the SPSS statistical package 11.5 (IBM, Armonk, NY, USA). The data were expressed as the mean \pm standard error of the mean. $\mathrm{P}<0.01$ and $\mathrm{P}<0.05$ denote significantly statistical differences.

\section{Results}

Immunohistochemical detection of Pdcd5. Formalin-fixed, paraffin-embedded specimens from 30 highly differentiated lung carcinoma and 20 healthy tissues were analyzed by immunohistochemistry in order to detect the protein expression of Pdcd5. As shown in Fig. 1, positive staining for Pdcd5 in healthy tissues was mainly observed in the cytoplasm, 

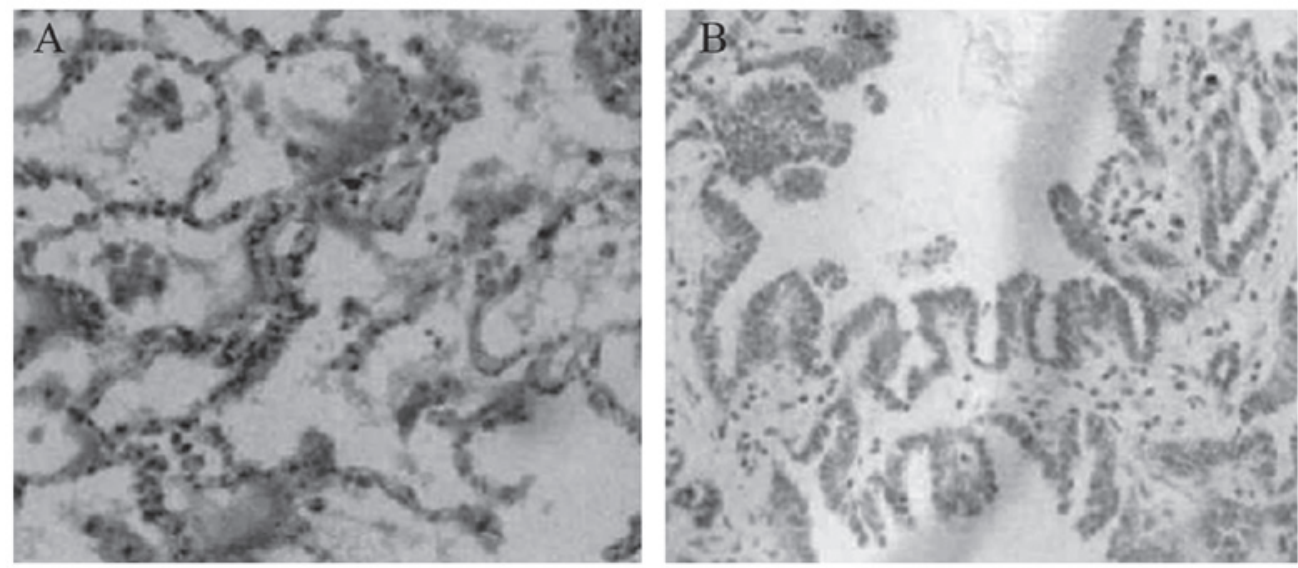

Figure 1. Paraffin-embedded samples of (A) healthy lung tissues and (B) highly differentiated lung adenocarcinoma tissues, analyzed by histochemical staining of the programmed cell death 5 (Pdcd5) protein and observed under a light microscope (magnification, x200). In healthy tissues, Pdcd5 is expressed in the cytoplasm, with some positive staining also detected in the nucleus. The cytoplasmic expression of Pdcd5 is decreased in adenocarcinoma tissues.

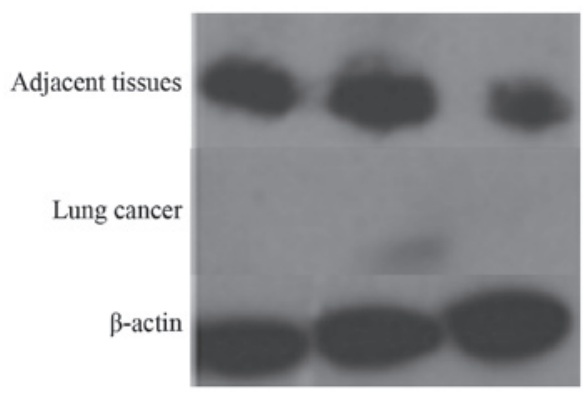

Figure 2. Western blot analysis of programmed cell death 5 (Pdcd5) in adjacent tissues and lung cancer samples. $\beta$-actin was used as the loading control. The blot is representative of three independent experiments.

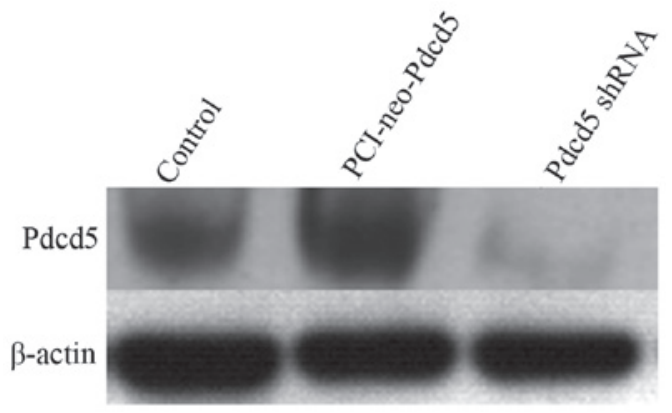

Figure 3. Western blotting aiming to analyze the efficiency of overexpression and silencing of the programmed cell death 5 (Pdcd5) gene, using A549 cell transfection with the PCI-neo-Pdcd5 and Pdcd5 short hairpin RNA (shRNA), respectively. The expression of Pdcd5 was detected by western blotting $48 \mathrm{~h}$ post-transfection, with $\beta$-actin was used as the loading control.

uniformly distributed, and in some cases, in the nucleus. By contrast, decreased immunoreactivity for Pdcd5 was observed in lung carcinoma tissues.

Western blot analysis of Pdcd5 expression. Next, we compared the expression level of Pdcd5 between lung carcinoma and healthy tissues by western blotting. As shown in Fig. 2, the results of three independent experiments showed that Pdcd5 expression is markedly decreased in lung cancer tissues compared to healthy ones.

The efficiency of overexpression and interference of Pdcd5. We next used the lung adenocarcinoma cell line A549 as a cell model to study the effects of $P d c d 5$ gene overexpression and silencing at the protein level by western blotting. $P d c d 5$ overexpression was achieved by transfecting A549 cells with the PCI-neo-Pdcd5 plasmid, and gene silencing by using a $P d c d 5$-specific shRNA. As shown in Fig. 3, the protein expression of Pdcd5 was markedly reduced in cells transfected with the shRNA, while a slight increase in the Pdcd5 level was observed in cells transfected with the PCI-neo-Pdcd5 plasmid.

Overexpression of Pdcd5 inhibits proliferation in the lung cancer cell line A549. The effects of Pdcd5 on cell viability and proliferation of lung cancer cells were assessed by the MTT and colony formation assays, respectively. As shown in Fig. 4, when Pdcd5 was overexpressed, a significant and time-dependent increase in A549 cell death was observed compared to untransfected cells $(\mathrm{P}<0.01)$. In addition, the number of colonies was significantly decreased in PCI-neo-Pdcd5-transfected cells in the colony formation assay. Taken together, these results indicate that overexpression of Pdcd5 significantly inhibits A549 cell proliferation and that Pdcd5 may act as a potential tumor suppressor.

Overexpression of Pdcd5 induces apoptosis of the lung cancer cell line A549. In order to examine whether the inhibition of proliferation in A549 cells overexpressing Pdcd5 is related to cell apoptosis, FACS analysis was performed. A549 cells transfected with PCI-neo-Pdcd5 or Pdcd5 shRNA were subjected to dual labeling with Annexin V-FITC and propidium iodide (PI). As shown in Fig. 4C, the apoptotic rate was significantly higher in the PCI-neo-Pdcd5 group $(25.8 \%)$ compared with the control $(3.6 \%)(\mathrm{P}<0.01)$.

Activated caspase-3 and-9, increased Bax and decreased $B c l-2$ levels in Pdcd5-overexpressing cells. To further explore the mechanism by which expression of Pdcd5 induces apop- 
A

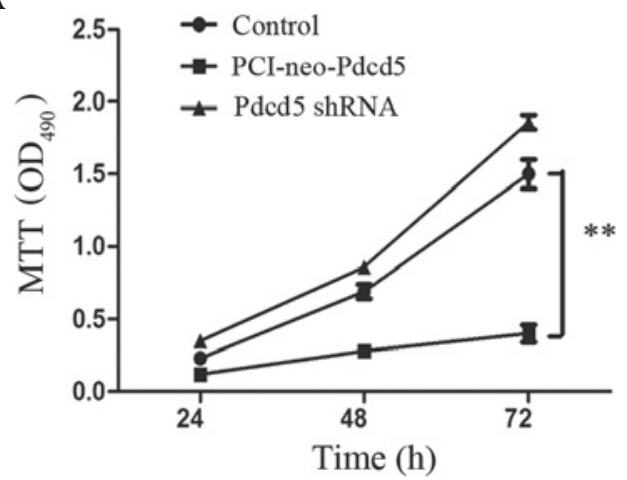

B

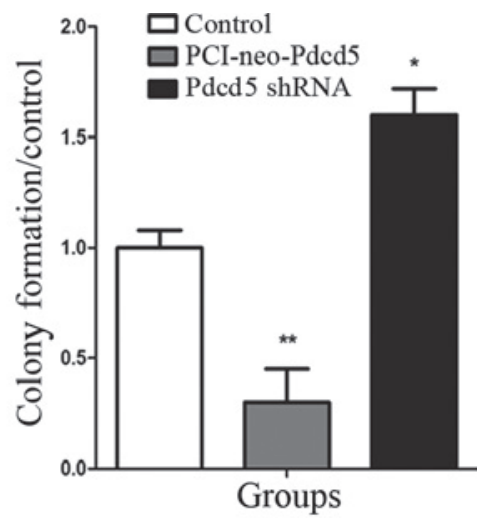

C
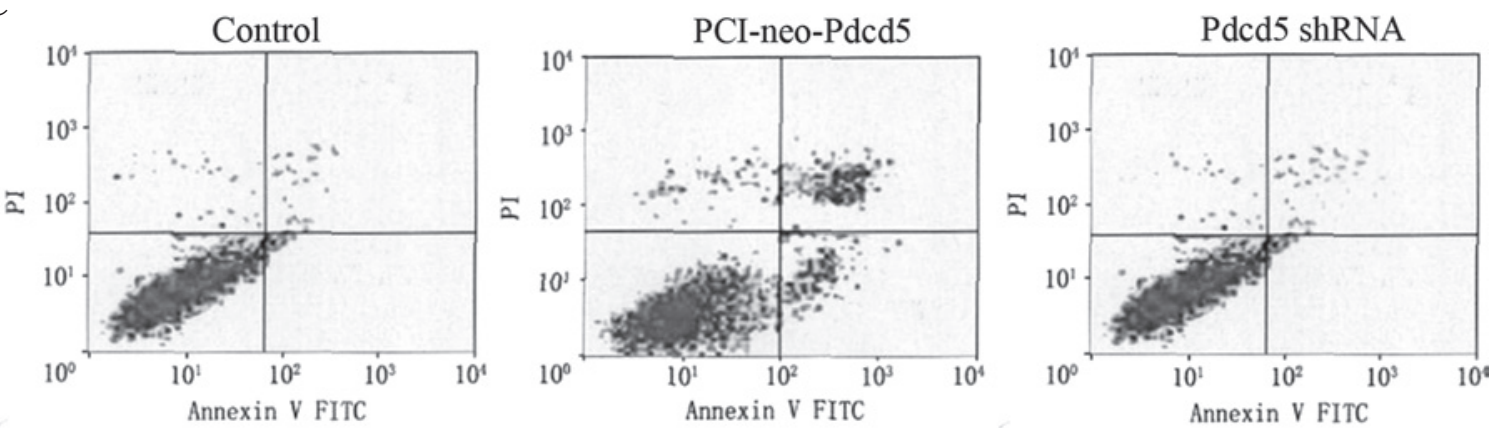

Figure 4. Overexpression of the programmed cell death 5 (Pdcd5) protein induces cell death, inhibits colony formation and promotes apoptosis in the lung cancer cell line A549. (A) MTT assay. A549 cells were transfected with PCI-neo-Pdcd5, Pdcd5 short hairpin RNA (shRNA) or were not transfected (control). After 24,48 and $72 \mathrm{~h}$, the optical density at $490 \mathrm{~nm}\left(\mathrm{OD}_{490}\right)$ was measured. ${ }^{* *} \mathrm{P}<0.01$, compared to the control. (B) Colony formation assay $48 \mathrm{~h}$ after ??? ${ }^{* *} \mathrm{P}<0.01,{ }^{*} \mathrm{P}<0.05$, compared to the control. (C) Apoptosis measured by flow cytometry. The cells of the three groups were stained with fluorescein isothiocyanate (FITC)-Annexin V and propidium iodide (PI). All experiments were repeated at least three times.

A

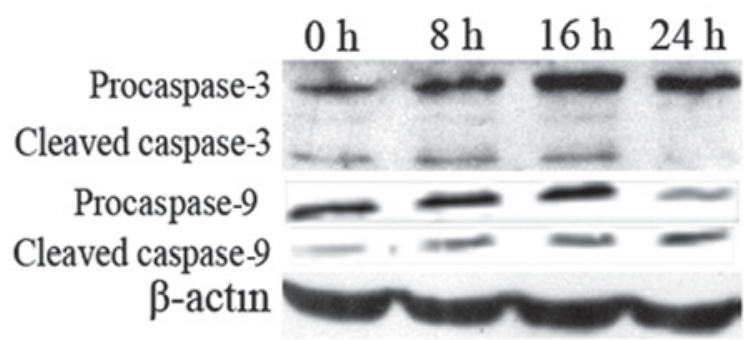

B

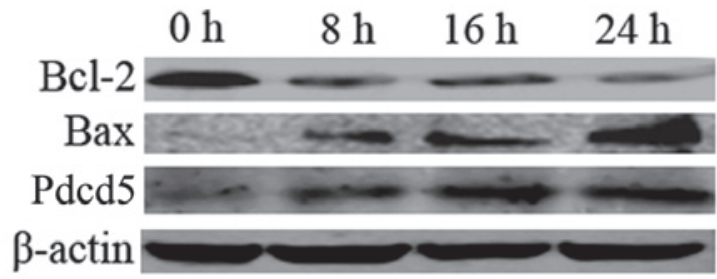

Figure 5. Western blot analysis of the expression of apoptosis-related proteins in A549 cells transfected with PCI-neo-Pdcd5 after 8 , 16 and 24 h. $\beta$-actin was used as a loading control. Expression of (A) pro- and cleaved caspase-3 and -9 and of (B) B-cell lymphoma 2 (Bcl-2), Bcl2-associated X protein (Bax) and programmed cell death $5(\operatorname{Pdcd} 5)$.

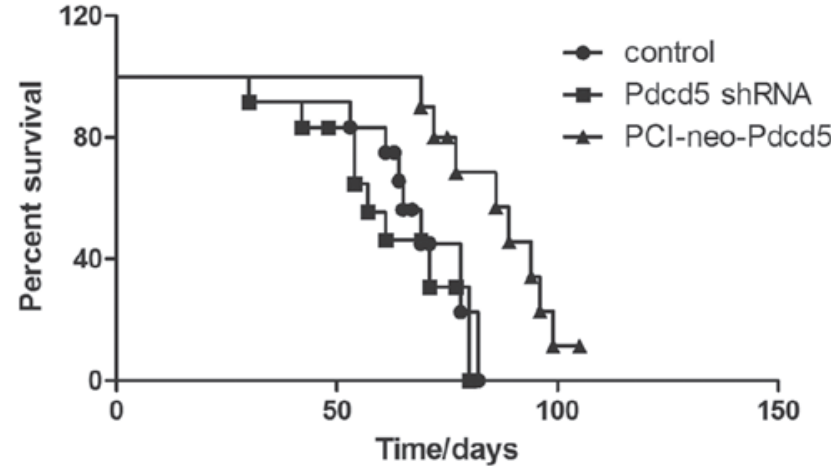

Figure 6. Survival rate of BalB/c mice following lethal challenge with lung carcinoma cells (A549 line). The mice were subcutaneously injected with $5 \times 10^{5}$ cells in their back. The survival rate was determined as $100 \mathrm{x}$ (number of survivors/total number of mice). Each group contained $>8$ mice. shRNA short hairpin RNA; control, mice injected with untransfected A549 cells. tosis, the levels of caspase-3, caspase-9 and Bcl-2 family proteins were examined by western blot analysis. As shown in Fig. 5A, both procaspase-3 and -9 were cleaved into their characteristic active forms, the relative level of which showed a time-dependent increase, suggesting that the intrinsic mitochondrial apoptotic pathway was activated. Moreover, the level of the $\mathrm{Bcl}-2$ protein was decreased and that of Bax was increased along with the increase in the Pdcd5 level in PCI-neo-Pdcd5-transfected cells (Fig. 5B).

Overexpression of Pdcd5 exhibits antitumor activity in a xenograft nude mouse model. In order to examine the ability of Pdcd5 to induce apoptosis in vivo, a nude mice xenograft model was established, and survival of mice injected with different types of A549 cells was calculated. As shown in Fig. 6, stable expression of Pdcd5 significantly $(\mathrm{P}<0.01)$ increased the 
survival rate of mice compared to Pdcd5 silencing or normal expression (control group).

\section{Discussion}

Lung cancer is one of the most common malignant tumor types in China. The incidence and mortality rates are rising every year. Imbalance between cell proliferation and apoptosis plays a vital role in the development of lung cancer, which prompted us to focus on the Pdcd5 protein. Pdcd5 is an apoptosis-regulated programmed cell death protein, first cloned in 1999 by Liu et al (14). The gene is widely expressed in various tissues, except for the hematopoietic system, and locates on chromosome 19q12-q1311 (27). The protein is composed of 125 amino acids, including 6 exons and 5 introns. Different expression levels of Pdc5 have been reported in various diseases, with reduced expression in leukemia (28), gastrointestinal stromal tumors (29), astrocytic gliomas (30) and prostate cancer (31). In the present study, the immunohistochemical analysis clearly showed positive staining of Pdcd5 in healthy lung tissues, mostly in the cell cytoplasm, and reduced staining in lung carcinoma tissues. This result was consistent with western blot analysis.

The PCI-neo-Pdcd5 plasmid was transfected into the human lung cancer cell line A549 to induce overexpression of Pdcd5. Consequently, apoptosis was induced in cancer cells, as detected by the MTT assay and flow cytometry analysis. It has been reported that Pdcd5 enhances cisplatin-induced apoptosis in chondrosarcomas $(32,33)$, which is consistent with findings from the present study. During the progression of apoptosis, the expression level of anti-apoptotic and proapoptotic proteins is tightly regulated. Here, the levels of Bcl-2 family proteins were detected by western blot analysis. The expression of Bax was increased and that of Bcl-2 was decreased after $24 \mathrm{~h}$ of transfection with the PCI-neo-Pdcd5 plasmid. The ratio of $\mathrm{Bax} / \mathrm{Bcl}-2$ was thus increased, and apoptosis is expected to be promoted in such conditions. In addition, the caspase- 3 and -9 were activated in A549 cells overexpressing Pdcd5, suggesting that Pdcd5 expression may activate the mitochondria-related apoptotic pathway.

In summary, our study analyzed the expression and clinical significance of Pdcd5 in lung cancer, but also provided evidence for the mechanism of PDCD5-induced cell apoptosis, showing that the mitochondria-related apoptotic signaling pathway may play an important role in the process. However, the exact molecular events of DCD5-induced cell apoptosis need to be explored in future studies. The present study indicated that Pdcd5 may be a useful target for the therapy of lung cancer.

\section{References}

1. Marshall HM, Bowman RV, Yang IA, Fong KM and Berg CD Screening for lung cancer with low-dose computed tomography: a review of current status. J Thorac Dis 5: S524-S539, 2013.

2. Lee PN and Forey BA: Indirectly estimated absolute lung cancer mortality rates by smoking status and histological type based on a systematic review. BMC Cancer 13: 189, 2013.

3. Amorin Kajatt E: Lung cancer: a review of current knowledge, diagnostic methods and therapeutic perspectives. Rev Peru Med Exp Salud Publica 30: 85-92, 2013 (In Spanish).

4. Centers for Disease Control and Prevention (CDC): State-specific trends in lung cancer incidence and smoking - United States, 1999-2008. MMWR Morb Mortal Wkly Rep 60: 1243-1247, 2011.
5. Jankovic M, Samarzija M, Jakopovic M, Kulis T and Znaor A: Trends in lung cancer incidence and mortality in Croatia, 1988-2008. Croat Med J 53: 93-99, 2012.

6. Kern DG, Kern E, Crausman RS and Clapp RW: A retrospective cohort study of lung cancer incidence in nylon flock workers, 1998-2008. Int J Occup Environ Health 17: 345-351, 2011.

7. Han R, Zheng R, Zhang S, Wu M and Chen W: Trend analyses on the differences of lung cancer incidence between gender, area and average age in China during 1989-2008. Zhongguo Fei Ai Za Zhi 16: 445-451, 2013 (In Chinese).

8. Chang S, Dai M, Ren JS, Chen YH and Guo LW: Estimates and prediction on incidence, mortality and prevalence of lung cancer in China in 2008. Zhonghua Liu Xing Bing Xue Za Zhi 33: 391-394, 2012 (In Chinese).

9. She J, Yang P, Hong Q and Bai C: Lung cancer in China: challenges and interventions. Chest 143: 1117-1126, 2013.

10. Huncharek M, Muscat J and Geschwind JF: K-ras oncogene mutation as a prognostic marker in non-small cell lung cancer: a combined analysis of 881 cases. Carcinogenesis 20: 1507-1510, 1999.

11. Suda K, Tomizawa K, Osada $\mathrm{H}$, et al: Conversion from the 'oncogene addiction' to 'drug addiction' by intensive inhibition of the EGFR and MET in lung cancer with activating EGFR mutation. Lung Cancer 76: 292-299, 2012.

12. You L, He B, Xu Z, et al: Inhibition of Wnt-2-mediated signaling induces programmed cell death in non-small-cell lung cancer cells. Oncogene 23: 6170-6174, 2004.

13. $\mathrm{Xu} \mathrm{L}, \mathrm{Hu}$ J, Zhao Y, et al: PDCD5 interacts with p53 and functions as a positive regulator in the p53 pathway. Apoptosis 17: 1235-1245, 2012

14. Liu H, Wang Y, Zhang Y, et al: TFAR19, a novel apoptosis-related gene cloned from human leukemia cell line TF-1, could enhance apoptosis of some tumor cells induced by growth factor withdrawal. Biochem Biophys Res Commun 254: 203-210, 1999.

15. Chen $\mathrm{CH}$, Jiang Z, Yan JH, et al: The involvement of programmed cell death 5 (PDCD5) in the regulation of apoptosis in cerebral ischemia/reperfusion injury. CNS Neurosci Ther 19: 566-576, 2013.

16. Gao F, Ding L, Zhao M, Qu Z, Huang S and Zhang L: The clinical significance of reduced programmed cell death 5 expression in human gastrointestinal stromal tumors. Oncol Rep 28: 2195-2199, 2012.

17. Zhuge C, Chang Y, Li Y, Chen Y and Lei J: PDCD5-regulated cell fate decision after ultraviolet-irradiation-induced DNA damage. Biophys J 101: 2582-2591, 2011.

18. Shi L, Song Q, Zhang Y, et al: Potent antitumor activities of recombinant human PDCD5 protein in combination with chemotherapy drugs in K562 cells. Biochem Biophys Res Commun 396: 224-230, 2010.

19. Saleem M, Maddodi N, Abu Zaid M, et al: Lupeol inhibits growth of highly aggressive human metastatic melanoma cells in vitro and in vivo by inducing apoptosis. Clin Cancer Res 14: 2119-2127, 2008.

20. Adhami VM, Siddiqui IA, Ahmad N, Gupta S and Mukhtar H: Oral consumption of green tea polyphenols inhibits insulinlike growth factor-I-induced signaling in an autochthonous mouse model of prostate cancer. Cancer Res 64: 8715-8722, 2004.

21. Ahmadian S, Barar J, Saei AA, Fakhree MA and Omidi Y: Cellular toxicity of nanogenomedicine in MCF-7 cell line: MTT assay. J Vis Exp: 26: e1191, 2009. doi: 10.3791/1191.

22. Verma A, Prasad KN, Singh AK, Nyati KK, Gupta RK and Paliwal VK: Evaluation of the MTT lymphocyte proliferation assay for the diagnosis of neurocysticercosis. J Microbiol Methods 81: 175-178, 2010.

23. Seidl K and Zinkernagel AS: The MTT assay is a rapid and reliable quantitative method to assess Staphylococcus aureus induced endothelial cell damage. J Microbiol Methods 92: 307-309, 2013.

24. Nishitani H, Sugimoto N, Roukos V, et al: Two E3 ubiquitin ligases, SCF-Skp2 and DDB1-Cul4, target human Cdt1 for proteolysis. EMBO J 25: 1126-1136, 2006.

25. Peng L, Xu Z, Zhou Y, Yang T, Liang ZQ and Zhang M: Effect of rosiglitazone on cells cycle, apoptosis and expression of Skp2 and p27Kip1 in hepatocellular carcinoma cell line. Zhonghua Gan Zang Bing Za Zhi 18: 148-149, 2010 (In Chinese).

26. Schulman BA, Carrano AC, Jeffrey PD, et al: Insights into SCF ubiquitin ligases from the structure of the Skp1-Skp2 complex. Nature 408: 381-386, 2000. 
27. Cheng A, Wang Y, Ma D, Zhou H and Lou S: Characterization of programmed cell death 5 (PDCD5) gene in human cartilage and its possible significance. Beijing Da Xue Xue Bao 35: 481-484, 2003 (In Chinese)

28. Ruan GR, Qin YZ, Chen SS, et al: Abnormal expression of the programmed cell death 5 gene in acute and chronic myeloid leukemia. Leuk Res 30: 1159-1165, 2006.

29. Li H, Wang Q, Gao F, et al: Reduced expression of PDCD5 is associated with high-grade astrocytic gliomas. Oncol Rep 20 573-579, 2008

30. Du YJ, Xiong L, Lou Y, Tan WL and Zheng SB: Reduced expression of programmed cell death 5 protein in tissue of human prostate cancer. Chin Med Sci J 24: 241-245, 2009.
31. Gao F, Ding L, Zhao M, Qu Z, Huang S and Zhang L: The clinical significance of reduced programmed cell death 5 expression in human gastrointestinal stromal tumors. Oncol Rep 28: 2195-2199, 2012.

32. Chen C, Zhou H, Xu L, et al: Recombinant human PDCD5 sensitizes chondrosarcomas to cisplatin chemotherapy in vitro and in vivo. Apoptosis 15: 805-813, 2010.

33. Yin A, Jiang Y, Zhang X, Zhao J and Luo H: Transfection of PDCD5 sensitizes colorectal cancer cells to cisplatin-induced apoptosis in vitro and in vivo. Eur J Pharmacol 649: 120-126, 2010. 